\title{
Is notch sensitivity a stress analysis problem?
}

\author{
Jaime Tupiassú Pinho de Castro, Marco Antonio Meggiolaro \\ Mechanical Engineering Department, Pontifical Catholic University of Rio de Janeiro (PUC-Rio), Brazil
}

\begin{abstract}
Semi-empirical notch sensitivity factors $q$ have been widely used to properly account for notch effects in fatigue design for a long time. However, the intrinsically empirical nature of this old concept can be avoided by modeling it using sound mechanical concepts that properly consider the influence of notch tip stress gradients on the growth behavior of mechanically short cracks. Moreover, this model requires only wellestablished mechanical properties, as it has no need for data-fitting or similar ill-defined empirical parameters. In this way, the $q$ value can now be calculated considering the characteristics of the notch geometry and of the loading, as well as the basic mechanical properties of the material, such as its fatigue limit and crack propagation threshold, if the problem is fatigue, or its equivalent resistances to crack initiation and to crack propagation under corrosion conditions, if the problem is environmentally assisted or stress corrosion cracking. Predictions based on this purely mechanical model have been validated by proper tests both in the fatigue and in the SCC cases, indicating that notch sensitivity can indeed be treated as a stress analysis problem.
\end{abstract}

KEYWORDS. Notch sensitivity; Stress gradient effects; Non-propagating short cracks.

\section{INTRODUCTION}

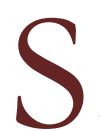
emi-empirical notch sensitivity factors $0 \leq q \leq 1$ have been long used to relate linear elastic (LE) stress concentration factors (SCF) $K_{t}=\sigma_{\max } / \sigma_{n}$, to their corresponding fatigue SCF $K_{f}=1+q \cdot\left(K_{t}-1\right)=S_{L}(\mathrm{R}) / S_{\text {Lnt }}(\mathrm{R})$, where $\sigma_{\max }$ and $\sigma_{\min }$ are the maximum and minimum LE stress at the notch root caused by $\sigma_{n}$, the nominal stress that would act at that point if the notch did not affect the stress field around the notch, whereas $S_{L}(R)$ and $S_{L \text { ntt }}(R)$ are the fatigue limits measured on standard (smooth and polished) and on notched test specimens (TS), respectively, at a given $R$ $=\sigma_{\min } / \sigma_{\max }$ ratio. Despite their empirical nature, such $K_{f}$ values are still widely used to quantify notch effects on the fatigue strength of structural components [1].

On the other hand, it is well known that the notch sensitivity in fatigue can be associated with the relatively fast generation of tiny non-propagating cracks at notch roots when $S_{L}(\mathrm{R}) / K_{t}<\sigma_{n}<S_{L}(\mathrm{R}) / K_{f}$, see Fig. 1 [2]. Based on this behavior, a model has been proposed to calculate the $q$ values from the fatigue behavior of short cracks emanating from notch tips, using only relatively simple but sound mechanical principles, which do not require heuristic arguments, neither any arbitrary data-fitting parameter [3-5]. In particular, this model does not require a critical distance concept, a good idea which originally assumed that the notch sensitivity should be dependent on some (yet never discovered) microstructural parameter [6-10]. In fact, to operationalize the critical distance concept it needs in practice to be fitted to the measured material properties, not to its microstructural parameters. The alternative explanation proposed here may be appealing for those who tackle such problems from a more mechanical viewpoint. Such an approach allowed it to explain notch effects not only on fatigue, but also on environmentally assisted cracking problems. Indeed, it is claimed here that notch sensitivity exists on such problems as well, and that it can be described using the same techniques successfully applied to model the fatigue problem. In fact, this can be done by just considering the proper resistances to crack initiation and to crack propagation in the given material/environment pair, both standard well-defined mechanical properties. Data is already available to support the proposed model predictions both in fatigue and SCC conditions. 


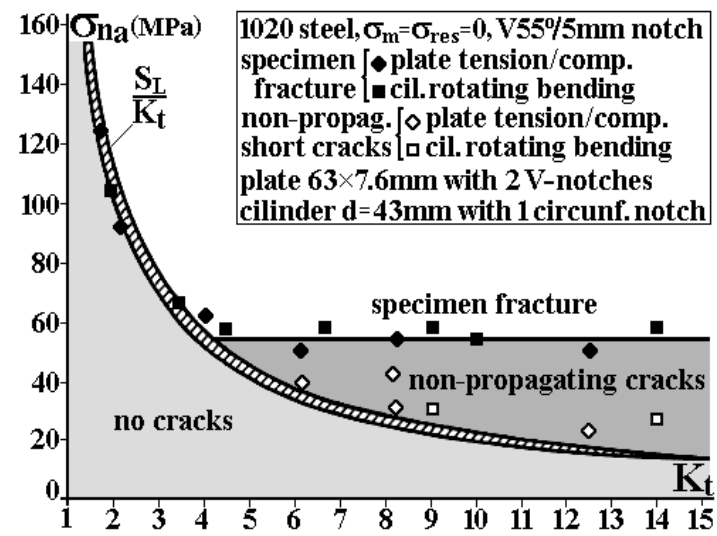

Figure 1: Classical data showing that non-propagating fatigue cracks are generated at the notch roots if $S_{L} / K_{t}<\sigma_{n}<S_{L} / K_{f}[2,7]$.

\section{ANALYSIS OF Notch EFFECTS ON THE FCG BEHAVIOR OF SHORT CRACKS}

$\mathrm{T}$ he generation of non-propagating fatigue cracks shown in Figure 1 indicates that whereas crack initiation by fatigue is controlled by the stresses acting at notch tips, the fatigue crack growth (FCG) behavior of short cracks emanating from them should be affected by the stress gradients around them too. It also indicates that notch sensitivity is caused by the existence of those non-propagating cracks. Indeed, to start a crack at a notch tip and then stop it after a short growth, its driving force must decrease in spite of its growing size. As the cracking driving force depends on the crack size and on the stress acting on it, the only mechanical explanation for such an apparently odd behavior is that the stresses ahead of the notch tip must decrease more than the crack growth contribution. Therefore, the stress gradients ahead of the notch tips must be as important as their SCF to understand the notch sensitivity behavior. Indeed, based on this sensible argument, even before developing its mechanics it could be claimed that, contrary to the traditional practice, for any given material the notch sensitivity value $q$ should depend not only on the notch tip radius $\rho$, but also on its depth $b$, since both affect the stress gradients around notch tips. This means that shallow and elongated notches of same $\rho$ may have quite different $q$. But before jumping to conclusions, let's work out this mechanical model.

First note that short fatigue cracks must behave differently from long cracks, since their FCG threshold must be smaller than the long crack threshold $\Delta K_{t h}(\mathrm{R})$ [11], otherwise the stress range $\Delta \sigma$ required to propagate them would be higher than the material fatigue limit $\Delta S_{L}(\mathrm{R})$. Indeed, assuming as usual that the FCG process is primarily controlled by the stress intensity factor (SIF) range, $\Delta K \propto \Delta \sigma \sqrt{ }(\pi a)$, if short cracks with $a \rightarrow 0$ had the same $\Delta K_{t b}(\mathrm{R})$ threshold of long cracks, their propagation by fatigue would require $\Delta \sigma \rightarrow \infty$, clearly a nonsense. Note also that the word "short" is used here to mean "mechanical" and not "microstructural" small cracks, since material isotropy is assumed in their modeling, a simplified hypothesis corroborated by the tests. The FCG threshold of short fatigue cracks under pulsating loads $\Delta K_{t b}(a, R=0)$ can be modeled using El Haddad-Topper-Smith (ETS) characteristic size $a_{0}$ [12], which is estimated from $\Delta S_{0}=\Delta S_{L}(\mathrm{R}=0)$ and $\Delta K_{0}=\Delta K_{t b}(\mathrm{R}=0)$. This clever trick reproduces the Kitagawa-Takahashi plot trend [13], see Fig. 2, using a modified SIF range $\Delta K^{\prime}$ to describe the fatigue propagation conditions of any crack, short or long, defined by:

$$
\Delta K^{\prime}=\Delta \sigma \sqrt{\pi\left(a+a_{0}\right)}, \text { where } a_{0}=(1 / \pi)\left(\Delta K_{0} / \Delta S_{0}\right)^{2}
$$

This $\Delta K$ ' has been deduced for the Griffith's plate SIF, $\Delta K=\Delta \sigma \sqrt{ }(\pi a)$. To deal with other geometries it should be rewritten using the non-dimensional geometry factor $g(a / w)$ of the cracked component:

$$
\Delta K^{\prime}=g(a / w) \cdot \Delta \sigma \sqrt{\pi\left(a+a_{0}\right)}, \text { where } a_{0}=(1 / \pi)\left[\Delta K_{0} /\left(g(a / w) \cdot \Delta S_{0}\right)\right]^{2}
$$

But the tolerable stress range $\Delta \sigma$ under pulsating loads only tends to the fatigue limit $\Delta S_{0}$ when $a \rightarrow 0$ if $\Delta \sigma$ is the notch root (instead of the nominal) stress range. However, most $g(a / w)$ expressions found in tables include the notch SCF, thus they use $\Delta \sigma$ instead of $\Delta \sigma_{n}$ as the nominal stress. A clearer way to define the short crack characteristic size $a_{0}$ when the short crack departs from a notch root is to explicitly recognize this practice, separating the geometry factor $g(a / w)$ into two 
parts: $g(a / w)=\eta \cdot \varphi(a)$, where $\varphi(a)$ describes the stress gradient ahead of the notch tip, which tends to the SCF as the crack length $a \rightarrow 0$, whereas $\eta$ encompasses all the remaining terms, such as the free surface correction:

$$
\Delta K^{\prime}=\eta \cdot \varphi(a) \cdot \Delta \sigma \sqrt{\pi\left(a+a_{0}\right)}, \text { where } a_{0}=(1 / \pi)\left[\Delta K_{0} /\left(\eta \cdot \Delta S_{0}\right)\right]^{2}
$$

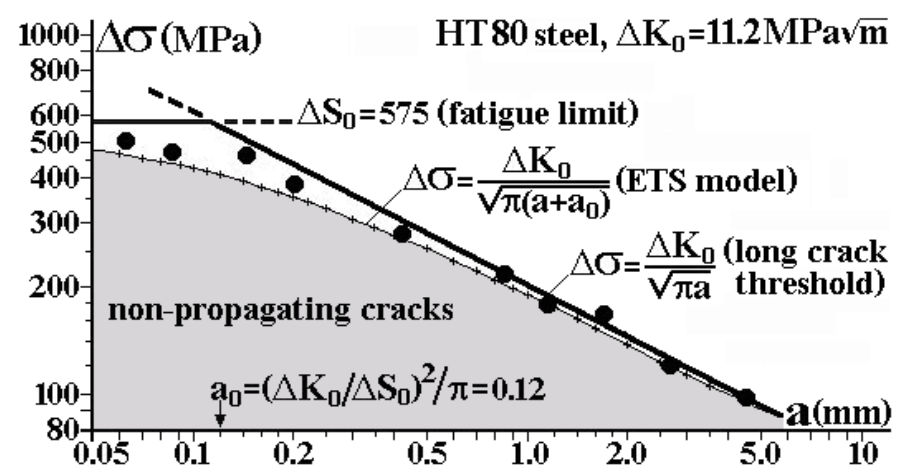

Figure 2: Kitagawa-Takahashi plot describing the fatigue propagation of short and long cracks under pulsating loads $(R=0)$ in $H$ T80 steel with $\Delta K_{0}=11.2 \mathrm{MPa} \sqrt{\mathrm{m}}_{\mathrm{m}}$ and $\Delta S_{0}=575 \mathrm{MPa}$.

Operationally, the short crack can be better treated by letting the SIF range retain its original form, while modifying the FCG threshold expression to become a function of the crack length $a$, namely $\Delta K_{0}(a)$, resulting (under pulsating loads) in:

$$
\Delta K_{0}(a)=\Delta K_{0} \cdot \sqrt{a /\left(a+a_{0}\right)}
$$

The ETS equation can be seen as one possible asymptotic match between the short and long crack behaviors. Following Bazant's idea [14], a more general equation can be used introducing an adjustable parameter $\gamma$ to fit experimental data:

$$
\Delta K_{0}(a)=\Delta K_{0} \cdot\left[1+\left(a_{0} / a\right)^{\gamma / 2}\right]^{-1 / \gamma}
$$

Equations (1) to (4) result from (5) if $\gamma=2$. The bi-linear limit, $\Delta \sigma\left(a \leq a_{0}\right)=\Delta S_{0}$ for short cracks and $\Delta K_{0}\left(a \geq a_{0}\right)=\Delta K_{0}$ for the long ones, is obtained if $g(a / w)=\eta \cdot \varphi(a)=1$ and $\gamma \rightarrow \infty$. Most short crack FCP data is fitted by $\Delta K_{0}(a)$ curves with $1.5 \leq \gamma \leq 8$, but $\gamma=6$ better reproduces classical $q$-plots based on data measured by testing semi-circular notched fatigue TS [3-5]. Using (5) as the FCP threshold, then any crack departing from a notch under pulsating loads should propagate if:

$$
\Delta K=\eta \cdot \varphi(a / \rho) \cdot \Delta \sigma \sqrt{\pi a}>\Delta K_{0}(a)=\Delta K_{0} \cdot\left[1+\left(a_{0} / a\right)^{\gamma / 2}\right]^{-1 / \gamma}
$$

where $\eta=1.12$ is the free surface correction. As fatigue depends on two driving forces, $\Delta \sigma$ and $\sigma_{\max }$, (6) can be extended to consider $\sigma_{\max }$ (indirectly modeled by the R-ratio) influence in short crack behavior. First, the short crack characteristic size should be defined using the fatigue limit $\Delta S_{R}$ and the FCP threshold for long cracks $\Delta K_{R}=\Delta K_{t b}\left(a>>a_{R}, R\right)$, both measured or properly estimated at the desired R-ratio:

$$
a_{\mathrm{R}}=(1 / \pi)\left[\Delta K_{\mathrm{R}} /\left(1.12 \cdot \Delta S_{\mathrm{R}}\right)\right]^{2}
$$

Likewise, the corresponding short crack FCP threshold should be re-written as:

$$
\Delta K_{\mathrm{R}}(a)=\Delta K_{\mathrm{R}} \cdot\left[1+\left(a_{\mathrm{R}} / a\right)^{\gamma / 2}\right]^{-1 / \gamma}
$$

According to Tada [15], the SIF of a crack with size $a$ that departs from a circular hole of radius $\rho$ given within $1 \%$ by:

$$
\left\{\begin{array}{l}
K_{I}=1.1215 \cdot \sigma \sqrt{\pi a} \cdot \varphi(x), x \equiv a / \rho \\
\varphi(x)=\left[1+\frac{0.2}{(1+x)}+\frac{0.3}{(1+x)^{6}}\right] \cdot\left[2-2.354\left(\frac{x}{1+x}\right)+1.206\left(\frac{x}{1+x}\right)^{2}-0.221\left(\frac{x}{1+x}\right)^{3}\right]
\end{array}\right.
$$


So, for circular holes $\varphi(x=0)=3$ and $\varphi(x \rightarrow \infty)=1 / 1.1215 \sqrt{2} \cong 0.63$. Since to propagate by fatigue any crack its SIF must be larger than its threshold, then for pulsating loads:

$$
\Delta K_{I}=\Delta \sigma \sqrt{\pi a} \cdot \eta \cdot \varphi(a / \rho)>\Delta K_{t b}(a)=\Delta K_{0} \cdot\left[1+\left(a_{0} / a\right)^{\gamma / 2}\right]^{-1 / \gamma}
$$

Note that $a_{0}$ cannot depend on the stress gradient factor $\varphi(a / w)$, otherwise it would not be a material property. The FCG criterion can thus be rewritten using two dimensionless functions, one related to the notch stress gradient $\varphi(a / w)$, and the other $g\left(\Delta S_{0} / \Delta \sigma, a / \rho, \Delta K_{0} / \Delta S_{0} \gamma \rho, \gamma\right)$ which includes the applied stress range $\Delta \sigma$, and depends on the crack size, on the notch radius $\rho$, on the fatigue resistances $\Delta K_{0}$ and $\Delta S_{0}$, and on the data fitting exponent $\gamma$ (if it is used):

$$
\varphi(a / \rho)>\frac{\left(\Delta S_{0} / \Delta \sigma\right) \cdot\left[\Delta K_{0} /\left(\Delta S_{0} \sqrt{\rho}\right)\right]}{\left[(\eta \sqrt{\pi a / \rho})^{\gamma}+\left[\Delta K_{0} /\left(\Delta S_{0} \sqrt{\rho}\right)\right]^{\gamma}\right]^{1 / \gamma}} \equiv g\left(\frac{\Delta S_{0}}{\Delta \sigma}, \frac{a}{\rho}, \frac{\Delta K_{0}}{\Delta S_{0} \sqrt{\rho}}, \gamma\right)
$$

Therefore, if $x \equiv a / \rho$ and $\kappa \equiv \Delta K_{0} / \Delta S_{0} \sqrt{ } \rho=\eta \cdot \sqrt{ }\left(\pi a_{0} / \rho\right)$, a fatigue crack departing from a Kirsch hole under pulsating loads grows whenever $\varphi(x) / g\left(\Delta S_{0} / \Delta \sigma, x, \kappa, \gamma\right)>1$. Figure 3 plots some $\varphi / g$ functions for several fatigue strength to loading stress range ratios $\Delta S_{0} / \Delta \sigma$ as a function of the normalized crack length $x$, assuming a small notch root radius compared to the short crack characteristic size with $\rho \cong 1.40 a_{0}$, and a material with $\kappa=1.5$ and $\gamma=6[5]$.

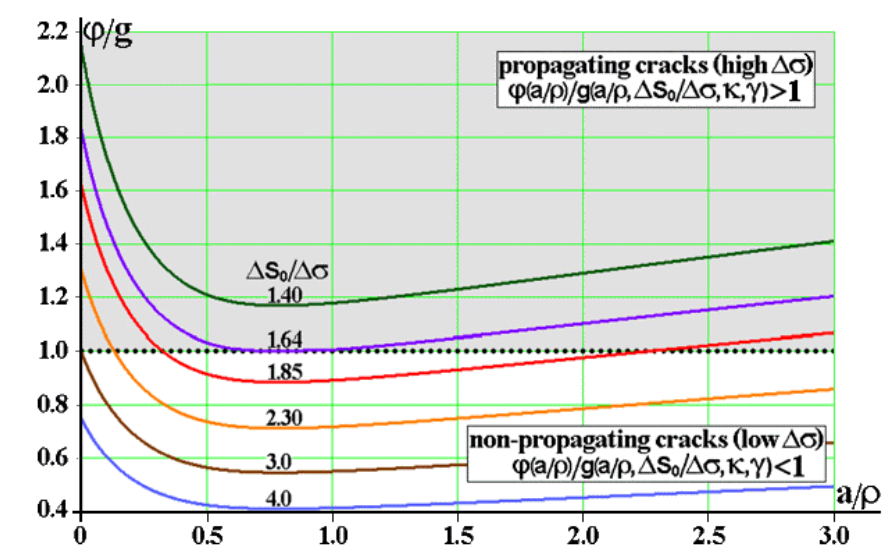

Figure 3: Cracks that can start from the border of a (small) circular hole may propagate by fatigue and then stop if their $\varphi / g<1$.

For high applied stress ranges $\Delta \sigma$, the strength to load ratio $\Delta S_{0} / \Delta \sigma$ is small, and the corresponding $\varphi / g$ curve is always higher than 1, so cracks initiate and propagate from this Kirsch hole border without stopping during this process. Small stress ranges with load ratios $\Delta S_{0} / \Delta \sigma \geq K_{t}=3$ have $\varphi / g<1$, meaning that such loads cannot initiate a fatigue crack from this hole, and that small enough cracks introduced there by any other means will not propagate at such low loads. Intermediate load ranges can initiate and propagate a fatigue crack from this hole border, until the decreasing $\varphi / g$ ratio reaches 1 , where the crack stops because the stress gradient ahead of its small root is sharp enough to eventually force $\Delta K_{I}(a)<\Delta K_{t h}(a)$. Finally, the curve is tangent to the $\varphi / g=1$ line identifies the smallest pulsating stress range that can cause crack initiation and propagation (without arrest) from the notch border by fatigue alone. Hence, by definition, it is associated to this hole fatigue SCF $K_{f}$. Moreover, the abscissa of its tangency point gives the largest non-propagating crack size that can arise from it by fatigue alone, $a_{\max }$. For any other $\rho / a_{0}, \gamma$, and $\kappa$ combination, $K_{f}$ and $a_{\max }$ can always be found by solving the system

$$
\left\{\begin{array} { l } 
{ \varphi / g = 1 } \\
{ \partial ( \varphi / g ) / \partial x = 0 }
\end{array} \Rightarrow \left\{\begin{array}{l}
\varphi\left(x_{\text {max }}\right)=g\left(x_{\text {max }}, K_{f}, \kappa, \gamma\right) \\
\partial \varphi\left(x_{\text {max }}\right) / \partial x=\partial g\left(x_{\text {max }}, K_{f}, \kappa, \gamma\right) / \partial x
\end{array}\right.\right.
$$

Kirsch holes induce relatively mild stress gradients. Larger holes compared with the short crack characteristic size, with radii $\rho>>a_{0}$, are associated to small $\kappa$ values and do not induce short crack arrest. That is a nice way to mechanically 
interpret the notch sensitivity concept. In other words, if the system $\{\varphi / g=1, \partial(\varphi / g) \partial x=0\}$ is solved for a given $\gamma$ and several tip radii using $\kappa \equiv \Delta K_{0} / \Delta S_{0} \sqrt{ } \rho$, then the notch sensitivity factor $q$ is obtained by:

$$
q(\kappa, \gamma) \equiv\left[K_{f}(\kappa, \gamma)-1\right] /\left(K_{t}-1\right)
$$

This approach has 4 advantages: (i) it is an analytical procedure; (ii) it considers the effect of the fatigue resistances to crack initiation and propagation on $q$; (iii) it can use the exponent $\gamma$ used to modify the original ETS model to better fit short FCG data; and (iv) it can be easily extended to other notch geometries. For example, the SIF of cracks that depart from a semi-elliptical notch with semi-axles $b$ and $c$, with $b$ collinear with the crack $a$ and perpendicular to the (nominal) stress $\sigma_{n}$, can be described by:

$$
\Delta K_{\mathrm{I}}=\eta \cdot F(a / b, c / b) \cdot \Delta \sigma \sqrt{\pi a}
$$

where $\eta=1.1215$ is the free surface correction factor and $F(a / b, c / b)$ is the geometrical factor associated to the notch stress concentration effect. Such notches SCF $K_{t}$ is given by [15]:

$$
K_{t}=(1+2 b / c) \cdot\left[1+0.1215 /(1+c / b)^{2.5}\right]
$$

Using $s=a /(a+b)$ two analytical expressions for $F(a / b, c / b)$ were obtained in [3] by fitting results obtained by a series of finite elements (FE) analyses for several types of semi-elliptical notches:

$$
\left\{\begin{array}{l}
F(a / b, c / b) \equiv f\left(K_{t}, s\right)=K_{t} \sqrt{\left[1-\exp \left(-K_{t}^{2} \cdot s\right)\right] /\left(K_{t}^{2} \cdot s\right)}, c \leq b \\
F(a / b, c / b) \equiv f^{\prime}\left(K_{t}, s\right)=K_{t} \sqrt{\frac{1-\exp \left(-K_{t}^{2} \cdot s\right)}{K_{t}^{2} \cdot s}} \cdot\left[1-\exp \left(-K_{t}^{2}\right)\right]^{-s / 2}, c \geq b
\end{array}\right.
$$

Note that traditional notch sensitivity estimates, like Peterson's $q=(1+\alpha / \rho)^{-1}$, where $\alpha$ is a length parameter obtained by fitting only 7 experimental points, suppose that the notch sensitivity depends only on the notch tip radius $\rho$ and on the steel tensile strength (and only on $\rho$ for $\mathrm{Al}$ alloys). The model proposed here, on the other hand, recognizes that $q$ depends on $\rho, \Delta S_{0}$, and $\Delta K_{0}$ (and in $\gamma$, if it is used). There are reasonable relations between $\Delta S_{0}$ and $S_{U}$, the ultimate tensile strength, but none between $\Delta K_{0}$ and $S_{U}$. This means that two steels of same $S_{U}$, but very different $\Delta K_{0}$, should behave identically according to Peterson-like $q$ estimates, for example, not a reasonable assumption. Further details on this model are available on [3], and experimental evidence that supports its predictions in fatigue is presented in [4-5]. Its extension for stress corrosion cracking conditions is developed in [16].

\section{INFLUENCE OF SHORT Cracks ON THE FATIGUE STRENGTH OF STRUCTURAL Components}

$\checkmark$ he traditional $S N$ and $\varepsilon N$ fatigue design methods are used to analyze supposedly crack-free pieces, but very often it is not possible to fulfill this requisite in practice. In fact, it is impossible to guarantee that a component is really free of cracks smaller than the detection threshold of the non-destructive method used to identify them. Nevertheless, most structural components are still designed against fatigue crack initiation using procedures that do not recognize such small cracks. Hence, their "infinite life" predictions may become unreliable when they are introduced by any means during manufacture or service, and not quickly detected and properly removed. But while large cracks may be easily detected and dealt with, small cracks may pass unnoticed, even in careful inspections. Therefore, structural components that must last for very long fatigue lives should be designed to be tolerant to undetectable short cracks, since continuous work under fatigue loads cannot be guaranteed if any of the cracks they might have can somehow propagate during their service lives.

Despite being self-evident, this requirement is still not included in most fatigue design routines used in practice. Indeed, most long-life designs just intend to maintain the service stresses at the structural component critical point below its fatigue limit, $\Delta \sigma<\Delta S_{L}(R) / \varphi_{F}$, where $\varphi_{F}$ is a suitable safety factor. Such calculations can become quite involved when designing e.g. against fatigue damage caused by random non-proportional loads, but their safe-life philosophy remains the same. However, in spite of not recognizing any cracks, most long-life designs work just fine. This means that they are somehow tolerant to undetectable or to functionally admissible short cracks. But the question "how much tolerant" 
cannot be answered by $S N$ or $\varepsilon N$ procedures alone. Such problem can be avoided by adding short crack concepts to their "infinite" life design criteria which, in its simplest version, may be given by [5]:

$$
\Delta \sigma<\Delta K_{\mathrm{R}} / \varphi_{F}\left\{\sqrt{\pi a} \cdot g(a / w) \cdot\left[1+\left(a_{\mathrm{R}} / a\right)^{\gamma / 2}\right]^{1 / \gamma}\right\}
$$

where $\Delta K_{\mathrm{R}}(a)=\Delta K_{\mathrm{R}} \cdot\left[1+\left(a_{\mathrm{R}} / a\right)^{\gamma / 2}\right]^{-1 / \gamma}$, and $a_{\mathrm{R}}=(1 / \pi)\left[\Delta K_{\mathrm{R}} / \eta \Delta S_{\mathrm{R}}\right]^{2}$

Since the fatigue limit $\Delta S_{R}=\Delta S_{L}(\mathrm{R})$ already reflects the effect of the microstructural defects inherent to the material, equation (17) complements it by describing the tolerances to small cracks that may pass unnoticed in actual service conditions. The practical usefulness of this sensible criterion is well illustrated by a practical example, as follows.

Due to a rare manufacturing problem, a batch of an important component left the factory with tiny surface cracks (only detectable by a microscope), causing some unexpected and embarrassing failures. Hence, it became necessary to properly quantify the actual effect of such tiny cracks in that component fatigue strength. Assume its rectangular cross-section has 2 by $3.4 \mathrm{~mm}$; its (uncracked) fatigue limit is $S_{L}=246 \mathrm{MPa}$; and it is made from steel with $S_{U}=990 \mathrm{MPa}$. This measured fatigue limit is about $1 / 4$ of the steel $S_{U} / 4$, whereas it would be traditionally estimated by $S_{L} \cong S_{U} / 2=495 M P a$. This difference may be due to a surface finish factor $k_{s f}=0.5$, a value between those proposed by Juvinall for $S_{U}=1 G P a$ steels with cold-drawn $\left(k_{s f}=0.45\right)$ and machined surfaces $\left(k_{s f}=0.7\right)$ [17]. The surface finish should not affect cracks, but as this difference could be due to other factors too, like tensile residual stresses, the only safe option is to use $S_{L}=246 M P a$ to evaluate the tiny crack effects. Therefore, by Goodman $S_{L}(\mathrm{R})=S_{R}=S_{L} S_{U}(1-R) /\left[S_{U}(1-R)+S_{L}(1+R)\right]$ for $R>-1$ (or $\left.\sigma_{m}>0\right)$, for example. The FCG threshold $\Delta K_{R}$ is also needed to model short crack effects, but if data is not available, as in this case, it must be estimated e.g. by $\Delta K_{R}(\mathrm{R} \leq 0.17)=\Delta K_{0}=6 M P a \sqrt{ } m$ and $\Delta K_{R}(\mathrm{R}>0.17)=7 \cdot(1-0.85 \mathrm{R})$ [18]. This practice increases the predictions uncertainty, but it is the only option available. Besides, it tends to be conservative. Moreover, it assumes that $\Delta K_{R}(R<0) \cong \Delta K_{0}$, a safe estimate too (except if the load history contained severe compressive underloads which might accelerate the crack, not the case here.) Using the SIF of an edge cracked strip of width $w$ loaded in mode I, then the tolerable stress ranges under pulsating loads shown in Fig. 4 are estimated within a fatigue safety factor $\varphi_{F}$ as:

$$
\Delta \sigma_{0} \leq \frac{\Delta K_{0} /\left(\varphi_{F} \sqrt{\pi a}\right)}{\left[0.752+2.02 \frac{a}{w}+0.37\left(1-\sin \frac{\pi a}{2 w}\right)^{3}\right] \sec \frac{\pi a}{2 w} \sqrt{\frac{2 w}{\pi a} \tan \frac{\pi a}{2 w}}\left[1+\left(\frac{a_{0}}{a}\right)^{\gamma / 2}\right]^{1 / \gamma}}
$$

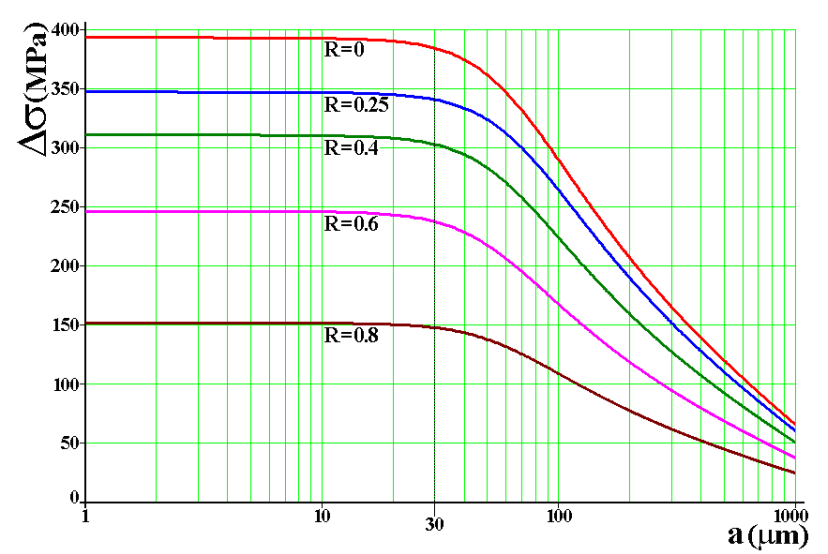

Figure 4: Larger stress ranges tolerable under several $\mathrm{R}$-ratios by the analyzed component considering it contains an edge crack with size $a$, for $w=3.4 m m, \eta=1.12, \Delta K_{0}=6 \mathrm{MPa} \sqrt{m}, a_{0}=59 \mu m, \gamma=6$, and $\varphi_{\mathrm{F}}=1.6$.

\section{Notch Sensitivity in EnVironmentally Assisted Cracking Problems}

$\mathrm{T}$

he behavior of materials on aggressive environments is an important problem for many industries, because the costs and specially the delivery times for special EAC-resistant alloys keep increasing. Major problems occur e.g. in the oil industry, where oil and gas fields can contain considerably amounts of $\mathrm{H}_{2} \mathrm{~S}$, and in the aeronautical 
industry, where very light structures must operate in saline environments. But environmentally assisted cracking problems have been treated so far by simplistic structural integrity assessment procedures based on an overly conservative policy of totally avoiding material-environment pairs susceptible to EAC conditions. When such conditions are unavoidable during the service life of the structural component in question, the standard solution is just to choose a nobler material to build it, one that is resistant or immune to crack initiation and propagation by EAC in the operational environment. Alternatively, the solution may be to recover the structural component surface with a suitable properly adherent and scratch resistant EAC-resistant coating. But in many cases there are no such coatings available in the market.

Such inflexible design criteria may be safe, but they can also be too conservative if the material is summarily disqualified when it may suffer EAC in the service environment, without considering any stress analysis issues. Such decisions may cause severe cost penalties. Indeed, even though EAC conditions may still be difficult to define, due to the number of metallurgical, chemical, and in particular mechanical variables that affect them, structural integrity assessment procedures should always be used on the design stage to define a maximum tolerable flaw size. In fact no crack can grow unless driven by a tensile stress, caused by the superposition of applied loads, residual stresses induced by previous loads or overloads, and maintenance or manufacturing procedures. Thus EAC cracks cannot be properly evaluated neglecting the stress and strain fields that may drive them.

But such uneconomical decisions can be avoided, since we already know how different the behavior of deep and shallow fatigue cracks is, and how it can be treated in structural design. Therefore, it is now possible to propose an extension of the proved criteria for accepting shallow fatigue cracks to environmentally assisted cracking problems, assuming they also present a notch sensitivity behavior that can be mechanically described. If cracks behave well under EAC conditions, then a Kitagawa-like diagram can be used to quantify tolerable stresses, using the material EAC resistances to define a "short crack characteristic size under EAC conditions" by:

$$
a_{0}=(1 / \pi) \cdot\left(K_{I E A C} / \eta \cdot S_{E A C}\right)^{2}
$$

In this way all corrosion features are assumed to be properly described by the resistance to crack propagation $K_{I E A C}$ and by the resistance to crack initiation $S_{E A C}$ under fixed stress conditions in the analyzed material-environment pair. In other words, this model supposes that the mechanical parameters that govern the environmentally assisted cracking problem behave analogously to the equivalent parameters $\Delta K_{t h}(\mathrm{R})$ and $\Delta S_{L}(\mathrm{R})$ that control the fatigue problem, see Fig. 5 .

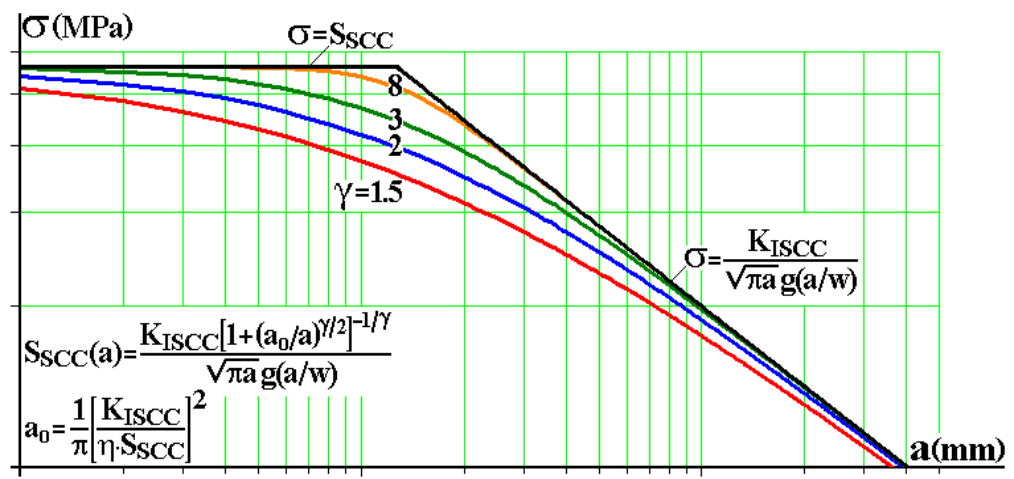

Figure 5: A Kitagawa-Takahashi-like plot proposed to describe the environmentally assisted cracking behavior of short and deep flaws for structural design purposes.

Consequently, if cracks loaded under EAC conditions behave mechanically as they should, i.e. if their driving force is indeed the stress intensity factor applied on them; and if the chemical effects that influence their behavior are completely described by the material resistance to crack initiation from smooth surfaces quantified by $\mathrm{S}_{\mathrm{EAC}}$, and by its resistance to crack propagation measured by $\mathrm{K}_{\mathrm{IEAC}}$; then it can be expected that EAC cracks may depart from sharp notches and then stop, due to the stress gradient ahead of the notch tips, eventually becoming non-propagating cracks, as it occurs in the fatigue case. Hence, if the size of non-propagating short cracks can be calculated using the same procedures useful for fatigue case, then the resistance to that kind of defect can be properly quantified using an EAC notch sensitivity factor in structural integrity assessments. Therefore, a criterion for the maximum tolerable crack size under EAC conditions can be proposed as:

$$
a_{\max } \leq(1 / \pi) \cdot\left[K_{I E A C}\left(1+a_{0} / a\right)^{\gamma / 2} / \sqrt{\pi a} \cdot g(a / w)\right]^{-1 / \gamma}
$$




\title{
CONCLUSIONS
}

\begin{abstract}
A generalized El Haddad-Topper-Smith's parameter was used to model the crack size dependence of the threshold stress intensity range for short cracks, as well as the behavior of non-propagating environmentally assisted cracks. This dependence was used to estimate the notch sensitivity factor $q$ of shallow and of elongated notches, from studying the propagation behavior of short non-propagating cracks that may initiate from their tips. It was found that the notch sensitivity of elongated slits has a very strong dependence on the notch aspect ratio, defined by the ratio $c / b$ of the semi-elliptical notch that approximates the slit shape having the same tip radius. These predictions were calculated by numerical routines. Based on this promising performance, a criterion to evaluate the influence of small or large surface flaws in fatigue and in environmentally assisted cracking problems was proposed. Such results indicate that notch sensitivity can indeed be properly treated as a mechanical problem.
\end{abstract}

\section{REFERENCES}

[1] Peterson, R.E., Stress Concentration Factors, Wiley (1974).

[2] Frost, N.E., Marsh, K.J., Pook, L.P., Metal Fatigue, Dover (1999).

[3] Meggiolaro, M.A., Miranda, A.C.O., Castro, J.T.P., Short crack threshold estimates to predict notch sensitivity factors in fatigue, Int. J. Fatigue, 29 (2007) 2022-2031.

[4] Wu, H., Imad, A., Nouredine, B., Castro, J.T.P., Meggiolaro, M.A., On the prediction of the residual fatigue life of cracked structures repaired by the stop-hole method, Int. J. Fatigue, 32 (2010) 670-677.

[5] Castro, J.T.P., Meggiolaro, M.A., Miranda, A.C.O., Wu, H., Imad, A., Nouredine, B., Prediction of fatigue crack initiation lives at elongated notch roots using short crack concepts, Int. J. Fatigue, 42 (2012) 172-182.

[6] Taylor, D., The Theory of Critical Distances: a New Perspective in Fracture Mechanics. Elsevier (2007).

[7] Taylor, D., Geometrical effects in fatigue: a unifying theoretical model, Int. J. Fatigue, 21 (1999) 413-420.

[8] Atzori, B., Lazzarin, P., Fillipi, S., Cracks and notches: analogies and differences of the relevant stress distributions and practical consequences in fatigue limit predictions, Int. J. Fatigue, 23 (2001) 355-362.

[9] Atzori, B., Lazzarin, P., Meneghetti, G., A unified treatment of the mode I fatigue limit of components containing notches or defects, Int J Fracture, 133 (2005) 61-87.

[10] Susmel, L., The theory of critical distances: a review of its applications in fatigue, Eng. Fract. Mechanics 75 (2008) 1706-1724.

[11] Lawson, L., Chen, E.Y., Meshii, M., Near-threshold fatigue: a review. Int. J. Fatigue, 21 (1999) 15-34.

[12] El Haddad, M.H., Topper, T.H., Smith, K.N., Prediction of non-propagating cracks. Eng. Fract. Mechanics, 11 (1979) 573-584.

[13] Kitagawa, H., Takahashi, S., Applicability of fracture mechanics to very small crack or cracks in the early stage. Proceedings of the $2^{\text {nd }}$ International Conference on Mechanical Behavior of Materials. ASM (1976) 627-631.

[14] Bazant, Z.P., Scaling of quasibrittle fracture: asymptotic analysis. Int. J. Fracture, 83 (1977) 19-40.

[15] Tada, H., Paris, P. C., Irwin, G. R., The Stress Analysis of Cracks Handbook, Del Research (1985).

[16] Castro, J.T.P., Leite, J.C.C., Does notch sensibility exist in environmentally assisted cracking (EAC)?, J. Mat. Research and Technology, in press, (2013).

[17] Juvinall, R.C., Marshek, K.M., Fundamentals of Machine Component Design, $4^{\text {th }}$ ed., Wiley (2005).

[18] Barsom, J.M., Rolfe, S.T., Fracture and Fatigue Control in Structures, 3 $3^{\text {rd }}$ ed., ASTM (1999). 